\title{
Shock-induced Amorphization in Covalently Bonded Solids
}

\author{
Shiteng Zhao ${ }^{1}$, Bimal $\mathrm{Kad}^{1}$, Eric $\mathrm{Hahn}^{1}$, Laura $\mathrm{Chen}^{2}$, Yekaterina, Opachi ${ }^{3}$, Karren More ${ }^{4}$, Bruce Remington ${ }^{5}$, Christopher \\ Wehrenberg ${ }^{5}$, Jerry LaSalvia ${ }^{6}$, Wen Yang ${ }^{1}$, Haocheng Quan ${ }^{1}$, and Marc Meyers ${ }^{1 *}$ \\ ${ }^{1}$ University of California, San Diego, La Jolla, CA 92093, USA \\ ${ }^{2}$ Oxford University, Oxford, OX1 2JD, UK \\ ${ }^{3}$ Nevada National Security Site, Livermore, CA 94550, USA \\ ${ }^{4}$ Oak Ridge National Laboratory, Oak Ridge, TN 21005, USA \\ ${ }^{5}$ Lawrence Livermore National Laboratory, Livermore, CA 94550, USA \\ ${ }^{6}$ U.S. Army Research Laboratory, MD 21005, USA
}

\begin{abstract}
Deposition of powerful pulsed laser energy onto a material, ablates its surface and drives a compressive shock wave propagating through it. Using this technique, unprecedented states of matter with extremely high pressures, temperatures, and strain rates can be experimentally studied. Here we report on laser-shock induced amorphization in four covalently bonded solids, namely silicon ( $\mathrm{Si}$ ), germanium (Ge), boron carbide $\left(\mathrm{B}_{4} \mathrm{C}\right)$ and silicon carbide $(\mathrm{SiC})$. Post shock transmission electron microscopy reveals that the newly formed amorphous materials exhibit a shear band alike morphology, suggesting that shear stress play a dominant role in this process. The density of these amorphous band decreases as a function of the distance to the surface and eventually disappeared at certain depth, which is coincident with the decay of the shock wave and indicates that there might be a critical stress for the onset of amorphization. Synchrotron XRay tomography of a recovered silicon target shows that large amounts of cracks are formed within the materials and the density also decrease with depth. Unlike amorphous bands, these cracks can propagate through the target, albeit without shattering the entire material. It is proposed that shock-induced amorphization is a new deformation mechanism of matter under extremely high rate deformation.
\end{abstract}

\section{Introduction}

When a shock wave passes through a crystalline solid, it compresses the materials and usually causes plastic deformation behind the shock front [1]. In ductile materials such as metals, this process is associated with generation of dislocations, and/or deformation twins, and/or stress-induced phase transformations [2]. For some extremely brittle materials, however, defectmediated plasticity is so limited that fracture seems to be the only way to dissipate the imposed strain energy, which normally leads to a catastrophic failure. Note that each of these mechanisms has a characteristic time scale and therefore is strongly strain rate-dependent. In many metals such as copper and tantalum, dislocations are the major carriers of plasticity at low strain rate whereas deformation twinning dominates at high strain rate, leading to the slip-twining transition [3]. As of fracture, it has been widely accepted that the propagation of crack is limited by Rayleigh wave speed [4]. Since the duration of the shock wave determines the strain rates, one may raise the question: what if the duration of the stress wave is orders of magnitude shorter than the required time for plasticity, fracture, and fragmentation?
Traditional shock wave methods such as gas gun and explosive driven techniques failed to fulfill this mission as they can only produce $\mu$ s stress pulses. Owing to the development of high power pulsed lasers, high amplitude (10s to 100 s of GPa) shock wave with ns pulse duration can be produced, which enables the exploration of materials science in this unknown regime. In this investigation, we have successfully shock recovered four different brittle materials, namely, $\mathrm{Si}$ [5-6], Ge [7], $\mathrm{B}_{4} \mathrm{C}$ [8], and $\mathrm{SiC}$, all of which are covalently bonded.

\section{Experimental Methods}

Laser shock experiments were carried out at Omega Laser Facility, Laboratory of Laser Energetics, University of Rochester, Jupiter Laser Facility, Lawrence Livermore National Laboratory.

We describe below a typical experimental configuration used in our shock recovery experiments, as shown in Fig. 1A. The high power pulsed laser irradiates onto a transparent ablator, usually a polymer with a very low density, ionizes it into rapidly expanding plasma that flows away from the energy deposition surface. Based on Newton's third law, the surface experience a reaction

* Corresponding author: mameyers@eng.ucsd.edu 
force equal to the rate of momentum that is carried away, leading to the establishment of shock wave that propagates radially through the ablator and eventually into the target materials.

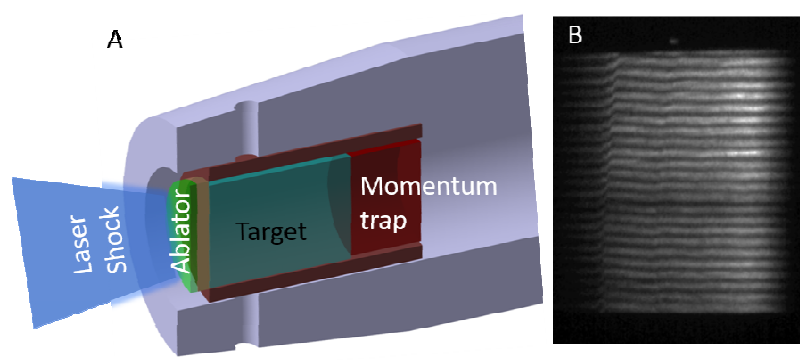

Fig. 1. Schematic drawing of the experimental set-up for the laser shock recovery experiments (A) and VISAR was used to determine the amplitude of the shock wave (B).

Such a process is usually termed as laser ablation and involves very complicated energy and mass transport. The ablation pressure is a function of laser parameters. An analytical scaling law was originally proposed by Lindl [9] and calibrated by various laser experiments,

$$
P(G P a)=(42 \pm 3) \cdot\left(\frac{I\left(T W / \mathrm{cm}^{2}\right)}{\lambda(\mu m)}\right)^{0.71 \pm 0.01}
$$

where $I=E / A t$ is the laser intensity, $\mathrm{A}, \mathrm{t}$ and $\lambda$ are the spot area, laser duration, and laser wavelength, respectively. Using modern large laser facility such as Omega, extraordinarily high pressures can be produced: using a $3.52 \mathrm{TW}\left(10^{12} \mathrm{~W}\right)$ laser with a wavelength of 352 $\mathrm{nm}$, the ablation pressure will be $\sim 210 \mathrm{GPa}$, which is almost the pressure inside the Earth's core $(\sim 350 \mathrm{GPa})$ ! It should also be mentioned here that not all of the laser energy can be absorbed; the coupling efficiency decreases as the wavelength of the laser increases. Velocity interferometer system for any reflector (VISAR) was used to determine the shock parameter and a typical VISAR data was shown in Fig. 1B.

In this investigation, we have studied four different covalently bonded solids. Silicon, germanium and silicon carbide are important semiconductors and therefore the high-purity crystals can be purchased commercially. We acquired these crystals with a purity of $99.99 \%$ from UniversityWafer Inc. The (001) orientation is chosen for silicon and germanium and (0001) orientation for silicon carbide. For boron carbide, the growth of monocrystalline materials is extremely difficult and not commercially available. Therefore, polycrystalline materials were used in this study, which are provided by the US Army Research Laboratory. Covalent bonds are typically strong, therefore all the four materials can be categorized as "hard materials". Ranking these materials in terms of hardness yields, $\mathrm{B}_{4} \mathrm{C}>\mathrm{SiC}>\mathrm{Si}>\mathrm{Ge}$. The applied laser parameters are summarized in Table 1 below. All targets are cylindrical in shape with a diameter of $3 \mathrm{~mm}$ and a height of $3 \mathrm{~mm}$.
Table 1 Laser Parameter Summary.

\begin{tabular}{|c|c|c|c|}
\hline Materials & $\begin{array}{c}\text { Laser } \\
\text { System } \\
\end{array}$ & $\begin{array}{c}\text { Key } \\
\text { Parameters }\end{array}$ & $\begin{array}{l}\text { Peak } \\
\text { Power } \\
\end{array}$ \\
\hline Silicon & Omega & $\begin{array}{l}1 \mathrm{~ns} \text { duration } \\
3 \mathrm{~mm} \text { spot }\end{array}$ & $\begin{array}{c}5.66 \\
\mathrm{TW} / \mathrm{cm}^{2}\end{array}$ \\
\hline $\begin{array}{c}\text { Boron } \\
\text { carbide/Silicon } \\
\text { carbide }\end{array}$ & Janus & $\begin{array}{c}3 \mathrm{~ns} \text { duration } \\
1 \mathrm{~mm}^{2} \text { spot }\end{array}$ & $\begin{array}{c}3.33 \\
\mathrm{TW} / \mathrm{cm}^{2}\end{array}$ \\
\hline Germanium & Omega & $\begin{array}{c}1 \mathrm{~ns} \text { duration } \\
3 \mathrm{~mm} \text { spot }\end{array}$ & $\begin{array}{c}2.83 \\
\mathrm{TW} / \mathrm{cm}^{2}\end{array}$ \\
\hline
\end{tabular}

\section{Results and Discussion}

Post-shock microstructural characterization was conducted to study the deformation/failure mechanism. $\mathrm{X}$-ray tomography reconstruction in Fig. 2 shows that the shock recovered target (silicon, shock pressure 20 GPa) contains a high density of cracks which propagate through the materials. A cross-section slice in Fig. 2B displays that the cracks near the surface has narrower interspacing than those away from it. A zoomed in view in Fig. 2C show that some cracks form clear crystallographic patterns. Two variants, along $\{111\}$ and $\{112\}$ planes, respectively, have been shown in Fig. 2C. Despite that large density of cracks were generated, they do not have sufficient time to fully develop and coalesce and thus the sample still maintains its integrity, which enables subsequent microstructural analysis.

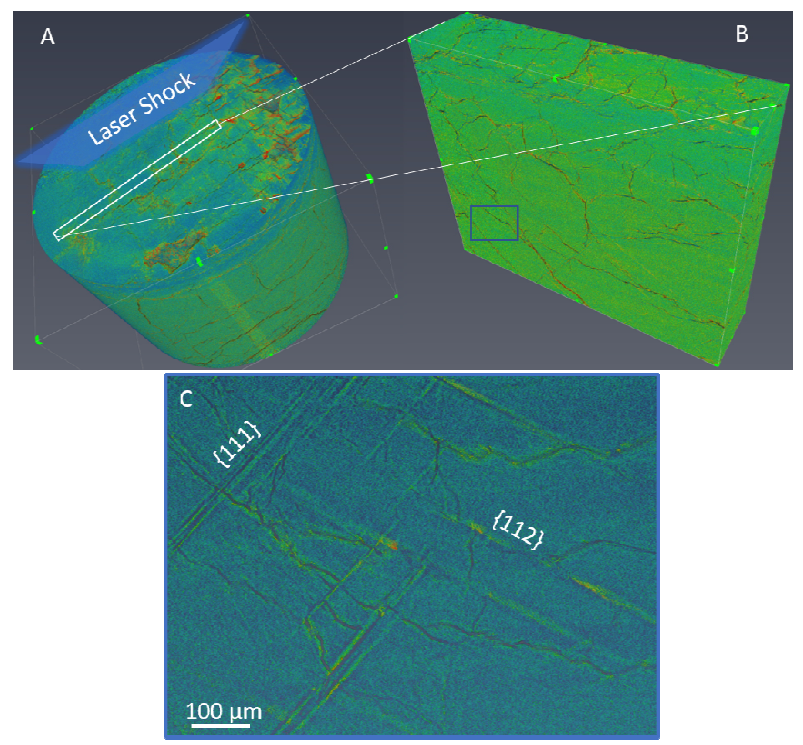

Fig. 2. Synchrotron X-ray computed tomography of the shock recovered target $(\mathrm{Si})$ shows a high density of cracks. (A)The cracks can propagate through the sample; however, the sample integrity is preserved. (B) A cross-section view shows that there is higher density of crack on the surface than that in the sample interior. (C) In addition to cracks, shear bands on $\{111\}$ and $\{112\}$ planes can be observed towards the bottom of the sample.

To understand the deformation mechanism at a finer scale, TEM analysis needs to be performed. We prepare the sample cite specifically on the shock surface by focused ion beam. Shear-band alike amorphous 
structures have been identified in all four materials, as seen in Fig. 3. The amorphous structure can be confirmed by the halo-ring shaped Fourier-transformed diffraction pattern in the inset 1 of Fig. 3A. For silicon (Fig. 3A, shock stress $\sim 20 \mathrm{GPa}$ ) and germanium (Fig. $3 \mathrm{~B}$, shock stress $\sim 10 \mathrm{GPa}$ ), in addition to the amorphous band, high density of stacking faults can be observed in the vicinity of the bands, suggesting that stacking faults are the precursors to amorphization. For boron carbide (Fig. 3C, shock stress $\sim 50 \mathrm{GPa}$ ) and silicon carbide (Fig. $3 \mathrm{D}$, shock stress $\sim 45 \mathrm{GPa}$ ), the interface is "free" from these crystalline defects. The minimum shock stress to observe amorphous band in these materials seems to scale with their strength, suggesting that the critical stress for amorphization depends on it.

The directional nature of the amorphous bands suggests that shear stress play a crucial role, triggering the crystalline-to-amorphous transition. Shear manifests itself in three possible ways: (1) shear strains cause massive inelastic lattice displacement that can lead to the loss of long-range order; (2) non-hydrodynamic stresses lower the melting temperature of materials significantly as the work done by shear stress provides the driving force for "Virtual Melting"[10]; (3) shear-induced plasticity causes localized heating which lead to localized thermal softening and reduce the mechanical barrier for amorphization. Specifically, the resolved shear stress on slip planes leads to the formation of stacking-faults in $\mathrm{Si}$ and $\mathrm{Ge}$, which are the precursors to amorphization. The deviatoric stresses are quite significant under shock compression up to the HEL, after which they will be relaxed by the directional amorphization. Since the initial material is crystalline, stress is a tensor and anisotropy play a very important role, especially in determining the path of the amorphization.

The temperature rise at the shock front is another crucial factor for amorphization. When materials undergo shock compression, their volume decreases, and consequently large work is imposed to the system. Therefore, this work is dissipated as heat and the temperature at the shock front rises accordingly. It is natural to conjecture that melting can occur at the shock front if the temperature rise exceeds the thermodynamic melting point of the materials. The negative Clapeyron slope for silicon, germanium, and boron carbide leads to a decrease in melting point with hydrostatic pressure, whereas the majority of materials exhibits an opposite trend. Therefore, it is easier for materials with negative Clapeyron slope to undergo shock-induced melting and amorphization.

\section{Conclusions}

In summary, we have shown that four covalently bonded materials undergo shock induced amorphization. It is therefore proposed that amorphization is a new deformation mechanism of these materials at ultrahigh strain rate $\left(>10^{7} / \mathrm{s}\right)$ when other mechanisms such as dislocation slip, twinning, phase transformation and fracture are kinetically less favorable. It should be mentioned that crack propagation was considered to be a post-shock effect and therefore it is fair to assume that amorphization occurs prior to fracture. The fact that most fracture planes are consistent with the orientations of the amorphous bands suggests that they are competing mechanisms. Therefore, the occurrence of amorphization can potentially suppress catastrophic failure of these brittle materials. The transformed amorphous structures can be re-crystallized, and the grain size can be tailored by controlling the subsequent thermal history, leading to a new route for microstructural engineering.
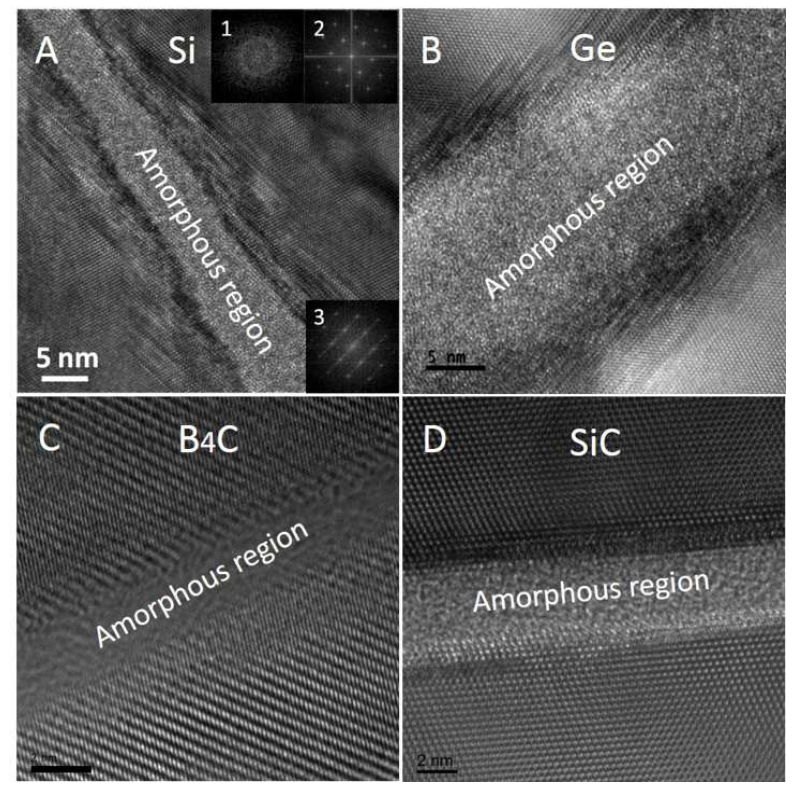

Fig. 3. High resolution TEM micrographs of amorphous bands in four different covalently bonded materials: (A) silicon, (B) Germanium, (C) Boron carbide, and (D) Silicon carbide. Note that high density of stacking faults were observed at the crystalline/amorphous interface in silicon and germanium whereas the interface of boron carbide and silicon carbide are relatively "defect free".

\section{References}

1. M.A. Meyers, Dynamic behavior of materials (John Wiley \& Sons, 1994)

2. B. Yaakobi, T.R. Boehly, D.D. Meyerhofer, T.J.B. Collins, B.A. Remington, P.G. Allen, S.M. Pollaine, H.E. Lorenzana, J.H. Eggert, EXAFS Measurement of Iron bcc-to-hep Phase Transformation in Nanosecond-Laser Shocks, Phys. Rev. Lett. 95 75501 (2005)

3. C.H. Lu, B.A. Remington, B.R. Maddox, B. Kad, H.S. Park, S.T. Prisbrey, M.A. Meyers, Laser compression of monocrystalline tantalum, Acta Mater. 60 66016620 (2012)

4. L.B. Freund, Crack propagation in an elastic solid subjected to general loading-II. Non-uniform rate of extension, J. Mech. Phys. Solids. 20 (1972) 141-152.

5. S. Zhao, B. Kad, E.N. Hahn, B.A. Remington, C.E. Wehrenburg, C.M. Huntington, M. Bringa, K.L. More, M.A. Meyers, Pressure and Shear Induced Amorphization of Silicon, EML. 5 74-80 (2015) 
6. S. Zhao, E.N. Hahn, B. Kad, B.A. Remington, C.E. Wehrenberg, E.M. Bringa, M.A. Meyers, Amorphization and nanocrystallization of silicon under shock compression, Acta Mater. 103 519-533 (2016)

7. S. Zhao, B. Kad, C.E. Wehrenberg, B.A. Remington, E.N. Hahn, K.L. More, M.A. Meyers, Generating gradient germanium nanostructures by shock-induced amorphization and crystallization, Proc. Natl. Acad. Sci. 114201708853 (2017)

8. S. Zhao, B. Kad, B.A. Remington, J.C. LaSalvia, C.E. Wehrenberg, K.D. Behler, M.A. Meyers, Directional amorphization of boron carbide subjected to laser shock compression, Proc. Natl. Acad. Sci. U.S.A. 113201604613 (2016)
9. J. Lindl, Development of the indirect-drive approach to inertial confinement fusion and the target physics basis for ignition and gain, Phys. Plasmas. 23933 (1995)

10. V.I. Levitas, R. Ravelo, Virtual melting as a new mechanism of stress relaxation under high strain rate loading., Proc. Natl. Acad. Sci. U. S. A. 109 13204-7 (2012) 